This item was submitted to Loughborough's Research Repository by the author.

Items in Figshare are protected by copyright, with all rights reserved, unless otherwise indicated.

\title{
A polynomial QR decomposition based turbo equalization technique for frequency selective MIMO channels.
}

PLEASE CITE THE PUBLISHED VERSION

PUBLISHER

(c) IEEE

VERSION

VoR (Version of Record)

\section{LICENCE}

CC BY-NC-ND 4.0

\section{REPOSITORY RECORD}

Davies, Martin, Sangarapillai Lambotharan, Joanne Foster, and Jonathon Chambers. 2019. "A Polynomial QR Decomposition Based Turbo Equalization Technique for Frequency Selective MIMO Channels.”. figshare. https://hdl.handle.net/2134/5554. 
This item was submitted to Loughborough's Institutional Repository (https://dspace.lboro.ac.uk/) by the author and is made available under the following Creative Commons Licence conditions.

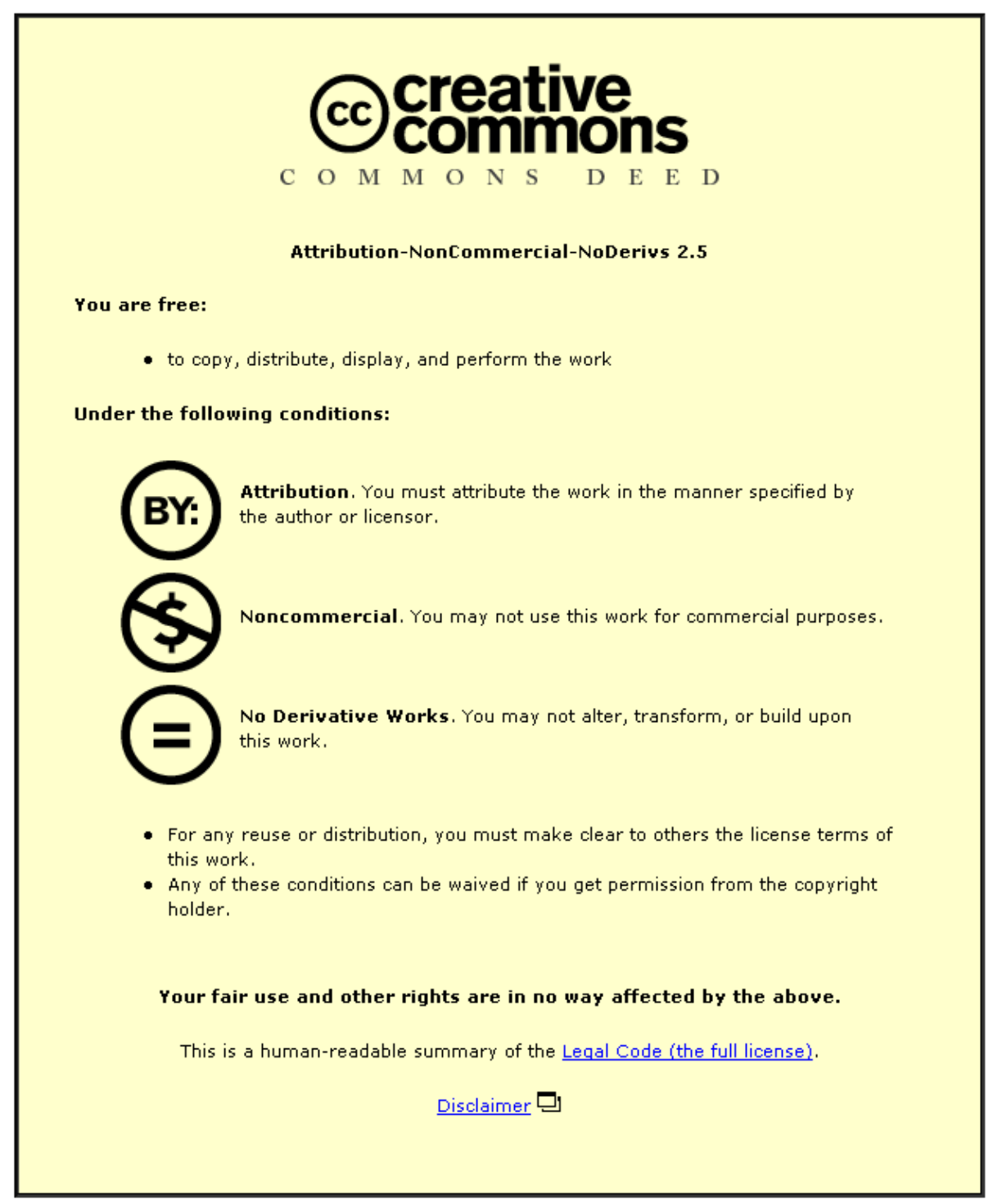

For the full text of this licence, please go to: http://creativecommons.org/licenses/by-nc-nd/2.5/ 


\section{A Polynomial QR Decomposition Based Turbo Equalization Technique for Frequency Selective MIMO Channels}

\author{
Martin Davies, Sangarapillai Lambotharan \\ Joanne Foster and Jonathon Chambers \\ Advanced Signal Processing Group \\ Department of Electronic and Electrical Engineering \\ Loughborough University LE11 3TU, UK \\ Email: M.R.Davies, S.Lambotharan, J.A.Foster, J.A.Chambers@lboro.ac.uk
}

\author{
John McWhirter \\ Center for D.S.P. \\ School of Engineering \\ Cardiff University CF24 3AA, UK \\ Email: McWhirterJG@cardiff.ac.uk
}

\begin{abstract}
In the case of a frequency flat multiple-input multiple-output (MIMO) system, QR decomposition can be applied to reduce the MIMO channel equalization problem to a set of decision feedback based single channel equalization problems. Using a novel technique for polynomial matrix $Q R$ decomposition (PMQRD) based on Givens rotations, we extend this work to frequency selective MIMO systems. A transmitter design based on Diagonal Bell Laboratories Layered Space Time (D-BLAST) encoding has been implemented. Turbo equalization is utilized at the receiver to overcome the multipath delay spread and to facilitate multi-stream data feedback. The effect of channel estimation error on system performance has also been considered to demonstrate the robustness of the proposed PMQRD scheme.

Average bit error rate simulations show a considerable improvement over a benchmark orthogonal frequency division multiplexing (OFDM) technique. The proposed scheme thereby has potential applicability in MIMO communication applications, particularly for TDMA systems with frequency selective channels.
\end{abstract}

\section{INTRODUCTION}

For a wireless system consisting of $M_{t}$ transmit antennas and $M_{r}$ receive antennas, the noise free channel can be represented as an $M_{r} \times M_{t}$ matrix, $\boldsymbol{H}$. In a frequency flat scenario where the received signals are instantaneously mixed, a scalar matrix is sufficient to describe the mixing. If the channel matrix is known to the receiver, its QR decomposition [1] can be formulated. Decomposing the channel matrix and exploiting the upper triangular structure of the resulting matrix, the set of source signals can be retrieved from the received signals using back substitution [2]. In the case of a frequency selective wireless system, the noise free multipath channel can be represented by a polynomial matrix, $\underline{\boldsymbol{H}}(z)$

$$
\underline{\boldsymbol{H}}(z)=\sum_{i=0}^{L-1} \boldsymbol{H}_{i} z^{-i}
$$

where $\boldsymbol{H}_{i} \in \mathbb{C}^{M_{r} \times M_{t}}$ is the $i^{t h}$ matrix tap of the MIMO channel of length $L$ and $z^{-i}$ is the unit delay operator. Thus its QR decomposition cannot be directly formulated. A typical approach to this broadband problem is to reduce it to a narrowband form by using a discrete Fourier transform (DFT) to split the broadband channel into narrow uniformly spaced frequency bands and applying the QR decomposition in each band. This is suitable for an OFDM based system. However in this paper, we propose a direct time domain based polynomial matrix decomposition as this is applicable to a range of access schemes such as time division multiple access (TDMA).

\section{A. Choice of Notation}

Throughout this paper, matrices are denoted by upper case bold characters and vectors by lower case bold characters. Regular upper or lower case characters denote scalar quantities. $[\cdot]_{k l}$ denotes the $(k, l)$-th element of the matrix in the square brackets. The superscripts $*, T$, and $H$ denote the complex conjugate, matrix transpose and Hermitian conjugate, respectively. $\boldsymbol{I}_{p}$ is used to denote the $p \times p$ identity matrix. Polynomial matrices and vectors are denoted by underscored bold upper and lower case characters, respectively. The use of an underscore with scalar quantities denotes a polynomial with scalar coefficients. Any polynomial (matrix, vector, or scalar) with the qualifier $(z)$ denotes a polynomial in the indeterminate variable $z^{-1}$. The $*$, used as a subscript, denotes complex conjugation of the coefficients in a polynomial matrix or vector. The use of $\sim$ above a polynomial matrix or vector denotes the paraconjugate, i.e. for a given polynomial matrix $\underline{\boldsymbol{A}}(z), \underline{\widetilde{\boldsymbol{A}}}(z)=\underline{\boldsymbol{A}}_{*}^{T}\left(z^{-1}\right) .\|\cdot\|_{F}$ will be used to denote the Frobenius norm (F-norm) of a polynomial matrix, which is simply the square root of sum of the squared F-norms for all coefficient matrices.

\section{Polynomial Matrix QR Decomposition}

The input to the PMQRD algorithm is a $p \times q$ complex polynomial matrix, $\underline{\boldsymbol{A}}(z)$.

$$
\underline{\boldsymbol{A}}(z)=\sum_{i=t_{1}}^{t_{2}} \boldsymbol{A}_{i} z^{-i}
$$

Where $\boldsymbol{A}_{i} \in \mathbb{C}^{p \times q}$ is the $i^{\text {th }}$ matrix tap of the polynomial matrix, $i \in \mathbb{Z}, t_{1}<t_{2}$ and $z^{i}$ is the unit delay operator. 
The polynomial QR decomposition of $\underline{\boldsymbol{A}}(z)$ is shown in (3) where $\boldsymbol{Q}(z)$ is a paraunitary polynomial matrix, such that $\underline{\boldsymbol{Q}}(z) \underline{\widetilde{\boldsymbol{Q}}}(z)=\boldsymbol{I}_{p}$ and $\underline{\boldsymbol{R}}(z)$ is an upper triangular polynomial matrix.

$$
\underline{\boldsymbol{A}}(z)=\underline{\boldsymbol{Q}}(z) \underline{\boldsymbol{R}}(z)
$$

The operation of the PMQRD algorithm is outside of the scope of the paper and is available in [3], [4].

\section{APPLICATION OF PMQRD to MIMO ChanNel EQUALIZATION}

Without loss of generality we consider a frequency selective MIMO system of equal number transmit and receive antennas, i.e. $M_{r}=M_{t}$. The PMQRD of the channel $\underline{\boldsymbol{H}}(z)$ is shown in (4).

$$
\underline{\boldsymbol{H}}(z)=\underline{\boldsymbol{Q}}(z) \underline{\boldsymbol{R}}(z)
$$

A set of source signals of length $N, \boldsymbol{s}(n) \in \mathbb{C}^{M_{t} \times 1}$ for $n \in$ $\{0,1, \ldots, N-1\}$ are propagated through the MIMO wireless channel, $\underline{\boldsymbol{H}}(z)$, received and filtered with $\underline{\widetilde{Q}}(z)$, as shown in Figure 1.

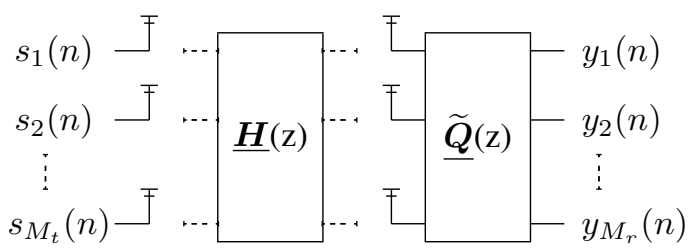

Fig. 1. PMQRD system diagram

$$
\boldsymbol{y}=\underline{\widetilde{\boldsymbol{Q}}}(z) \underline{\boldsymbol{H}}(z) \boldsymbol{s}+\underline{\widetilde{\boldsymbol{Q}}}(z) \boldsymbol{n}
$$

where $\boldsymbol{n}$ denotes an additive Gaussian noise process with variance $\sigma^{2} \boldsymbol{I}_{M_{r}}$. The convolutive mixing model can be rewritten as (6), where $\boldsymbol{n}^{\prime}=\underline{\widetilde{Q}}(z) \boldsymbol{n}$.

$$
\boldsymbol{y}=\underline{\boldsymbol{R}}(z) \boldsymbol{s}+\boldsymbol{n}^{\prime}
$$

\section{A. Iterative Interference Cancellation}

The MIMO channel problem can now be transformed into a set of $M_{r}$ equalization problems using back substitution. The $M_{t}^{t h}$ source signal is expressed as (7) which is a single channel equalization problem, which is solved using a minimum mean squared error (MMSE) equalizer [5].

$$
\boldsymbol{y}_{M_{r}}=\underline{\boldsymbol{r}}_{M_{r} M_{r}}(z) \boldsymbol{s}_{M_{t}}+\boldsymbol{n}_{M_{r}}^{\prime}
$$

Once $s_{M_{r}}$ is retrieved we use it to cancel its contribution to $\boldsymbol{y}_{M_{r}-1}$ as follows

$$
\boldsymbol{y}_{M_{r}-1}-\underline{\boldsymbol{r}}_{M_{r}-1 M_{r}}(z) \boldsymbol{s}_{M_{t}}=\underline{\boldsymbol{r}}_{M_{r}-1 M_{r}-1}(z) \boldsymbol{s}_{M_{t}-1}+\boldsymbol{n}_{M_{r}-1}^{\prime}
$$

which again is a single channel equalization problem. Therefore the $i^{t h}$ single channel equalization problem can be formulated as

$$
\boldsymbol{y}_{i}-\sum_{j=i+1}^{M_{r}} \underline{\boldsymbol{r}}_{i j}(z) \boldsymbol{s}_{j}=\underline{\boldsymbol{r}}_{i i}(z) \boldsymbol{s}_{i}+\boldsymbol{n}_{i}^{\prime}
$$

providing the streams $s_{i+1} \ldots s_{M_{t}}$ have been previously recovered.

\section{Channel Model}

In our simulations, we consider a MIMO system with three antennas at the transmitter and receiver. The temporal length, $L$ of the channel between each transmitter and receiver is five. The channel has a constant power delay profile with equal average gain for each tap.

\section{Diagonal Encoding (D-BLAST)}

D-BLAST [6] is an encoding architecture that combines the simplicity of horizontal BLAST (H-BLAST) encoding with the performance benefits of vertical BLAST (V-BLAST) encoding [7]. The data stream is first demultiplexed into three substreams, $s_{1}, s_{2}, s_{3}$. Each substream is then independently convolutionally encoded and interleaved. We have used the code formatting polynomials in (10) as per the global system for mobile (GSM) CS1-CS3 [8].

$$
\begin{aligned}
& G 0=1+D^{3}+D^{4} \\
& G 1=1+D^{1}+D^{3}+D^{4}
\end{aligned}
$$

To ensure that errors appear random and to avoid long error bursts, an interleaver is used to randomize the encoded bits prior to transmission. We have used an S-Random interleaver with a depth of 28 bits to gain maximum performance [9]. The data streams are then rotated, so that the bit stream-antenna association is periodically cycled [2]. This allows a diversity gain of $M_{r} M_{t}$ while maintaining a low computational complexity at the receiver.

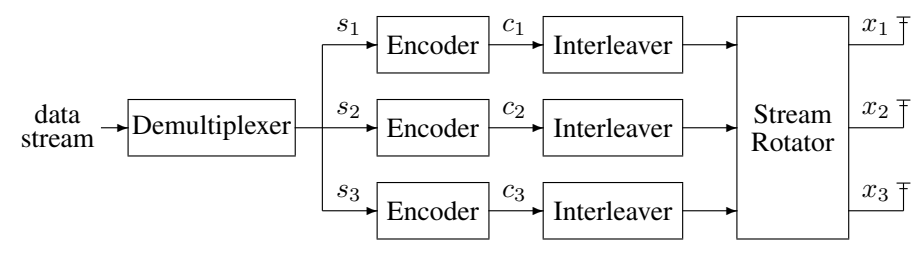

Fig. 2. D-BLAST transmitter architecture

\section{RECEIVER DESIGN}

The received signals are filtered with $\underline{\widetilde{Q}}(z)$ as shown in Figure 1, yielding the received substreams, $y_{1}, y_{2}, y_{3}$. Each substream is turbo equalized prior to the application of the iterative interference cancellation scheme previously described.

Turbo equalization [10] is an iterative equalization and decoding technique that can achieve impressive performance 
gains for coded data transmission over intersymbol interference (ISI) channels. Repeating the equalization and decoding tasks on the same set of data and incorporating soft feedback from the decoder into the equalization process generally yields significant improvements in the BER [10]. We assume the channel coefficients of the $i^{t h}$ stream, $\underline{\boldsymbol{r}}_{i i}(z)$ are known to the receiver and do not vary in time within each block. Figure 3 shows the turbo equalization structure.

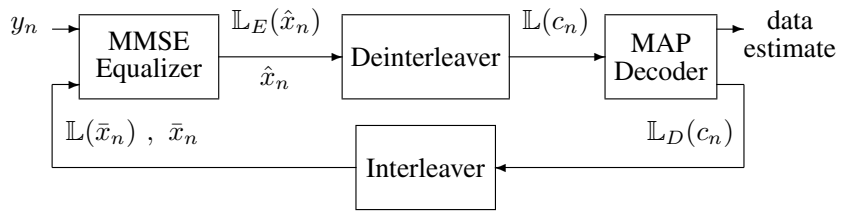

Fig. 3. Turbo equalization structure

The $\mathbb{L}$ operator is applied to quantities $x \in\{+1,-1\}$ and is given by

$$
\mathbb{L}(x)=\ln (P(x=+1) / P(x=-1))
$$

i.e., the log likelihood ratio (LLR).

\section{A. MMSE Equalizer}

The MMSE equalizer computes estimates $\hat{x}_{n}$ of the transmitted symbols $x_{n}$ from the received symbols $y_{n}$ by minimizing the cost function $E\left\{\left|x_{n}-\hat{x}_{n}\right|^{2}\right\}$ [5] where $\hat{x}_{n}$ represents the soft output from the MMSE equalizer, and $E\{\cdot\}$ denotes the statistical expectation operator.

For a single channel $\underline{\boldsymbol{r}}_{i i}(z)$ the channel is denoted as $h_{0}+h_{1} z^{-1}, \ldots, h_{L-1} z^{-(L-1)}$ where $L$ represents the channel length. We only consider taps of $\underline{\boldsymbol{r}}_{i i}(z)$ that are greater than $\sigma^{2} /\left(M_{r} M_{t}\right)$ where $\sigma^{2}$ represents the noise variance. We set the length of the equalizer, $M$, to capture these taps of interest. The channel convolution matrix, $\boldsymbol{H} c$ of dimensions $M \times(M+L-1)$ takes the form:

$$
\boldsymbol{H} c=\left[\begin{array}{ccccccc}
h_{0} & h_{1} & \ldots & h_{L-1} & 0 & \ldots & 0 \\
0 & h_{0} & h_{1} & \ldots & h_{L-1} & \ldots & 0 \\
& & & \ddots & \ddots & \ddots & \ddots \\
0 & \ldots & 0 & h_{0} & h_{1} & \ldots & h_{L-1}
\end{array}\right]
$$

Let $\boldsymbol{H} c_{M}$ denote the column of $\boldsymbol{H} c$ containing the most energy. Assuming symbols are temporally uncorrelated, we write $E\left\{\left(\boldsymbol{x}_{n}-\overline{\boldsymbol{x}}_{n}\right)\left(\boldsymbol{x}_{n}-\overline{\boldsymbol{x}}_{n}\right)^{H}\right\}$ as a diagonal matrix $\operatorname{diag}(\boldsymbol{v})$ where the $n^{\text {th }}$ element of $\boldsymbol{v}$ is $\boldsymbol{v}_{n}=1-\bar{x}_{n}^{2}$ and $\bar{x}_{n}$ denotes the interleaved soft estimates of the transmitted symbol from the MAP decoder output. The MMSE weight vector, $\boldsymbol{w}_{n}$ is given by [10] [5]:

$$
\boldsymbol{w}_{n}=\left(\boldsymbol{H} \boldsymbol{c} \times \operatorname{diag}\left(\boldsymbol{v}_{n}\right) \times \boldsymbol{H} c^{H}+\sigma^{2} \boldsymbol{I}\right)^{-1} \boldsymbol{H} c_{M}
$$

The MMSE equalizer output $\hat{x}_{n}$ is used to obtain the difference between the posteriori and a prior LLR as follows:

$$
\begin{aligned}
\mathbb{L}_{E}\left(\hat{x}_{n}\right) & =\ln \frac{p\left\{x_{n}=+\left.1\right|_{\hat{x}_{n}}\right\}}{p\left\{x_{n}=-\left.1\right|_{\hat{x}_{n}}\right\}}-\ln \frac{p\left\{x_{n}=+1\right\}}{p\left\{x_{n}=-1\right\}} \\
& =\ln \frac{p\left\{\left.\hat{x}_{n}\right|_{s_{n}=+1}\right\}}{p\left\{\left.\hat{x}_{n}\right|_{s_{n}=-1}\right\}} \\
& =\frac{4 \Re\left\{\hat{x}_{n}\right\}}{1-\boldsymbol{H} c_{M}^{T} \boldsymbol{w}_{n}}
\end{aligned}
$$

where $\Re\left\{\hat{x}_{n}\right\}$ denotes the real component of the quantity $\left\{\hat{x}_{n}\right\}$.

\section{B. MAP Decoder}

The maximum a posteriori (MAP) algorithm [11] computes the posterior probability of symbols from Markov sources transmitted through discrete memoryless channels. Since the output of a convolutional coder passed through the equalized frequency selective channel forms a Markov source the MAP algorithm can be used for maximum a posteriori probability decoding of convolutionally encoded code [12]. For each transmitted symbol it generates a hard estimate (using thresholding) and soft outputs, $\mathbb{L}_{D}\left(c_{n}\right), \bar{x}_{n}$ in the form of the a posteriori probability of the received sequence [13].

\section{Optimal Detection Ordering}

The performance of the iterative interference cancellation scheme is affected by the order in which the components of $x$ are detected. An optimal detection ordering scheme (ODO) scheme can significantly improve system performance. This is achieved by swapping the columns of $\underline{\boldsymbol{H}}(z)$ and performing the PMQRD. A permutation of the columns of $\underline{\boldsymbol{H}}(z)$ exists such that $\left\|\boldsymbol{r}_{3,3}\right\|_{F}$ is maximal. Wolniansky et al have shown [7] that the column permutation of $\underline{\boldsymbol{H}}(z)$ where maximising $\left\|\boldsymbol{r}_{i+1, i+1}\right\|_{F}$ given that $\left\|\boldsymbol{r}_{i, i}\right\|_{F}$ is already maximal yields the order of optimum detection.

\section{RESULTS}

We consider a wide sense quasi stationary (WSQS) situation where the channel coefficients have been assumed to be unchanged within each data block, but allowed to change between data blocks according to a zero mean complex circular Gaussian distribution. WSQS implies that the second-order time statistics of the channel are stationary and is justified in mobile channels over short periods [2]. The bit error rate has been computed for 1000 Monte Carlo simulations. The modulation scheme used is BPSK for evaluation purposes but extension to large constellations is straightforward. The number of time slots of the channel, $N=2048$. Initially we assume the receiver has perfect channel knowledge.

We have used a MIMO-OFDM QR scheme as a benchmark. MIMO-OFDM is a DFT based technique that decomposes the otherwise frequency selective channel of bandwidth $B$ into $N$ orthogonal frequency flat MIMO channels, each with a bandwidth $B / N$ [2]. The data stream undergoes the same encoding process as the PMQRD based scheme. Prior to transmission the transmitter performs an inverse fast Fourier transform (IFFT) operation on the signal to be transmitted 
from each individual transmit antenna. A cyclic prefix $(\mathrm{CP})$ of length $(L-1)$ is then added prior to transmission. At the receiver the cyclic prefix is stripped off and an FFT is applied to the signal received at each antenna. The standard $\mathrm{QR}$ decomposition is then applied within each narrowband tone. The iterative cancellation within the receiver is performed on each tone individually and Viterbi algorithm is used in the error correcting decoder. OFDM transmission incurs on average a loss in spectral efficiency of $(L-1) /(N+L-1)$ on account of the cyclic prefix. If $N \gg L$, this loss is negligible [2] so this has not been considered.

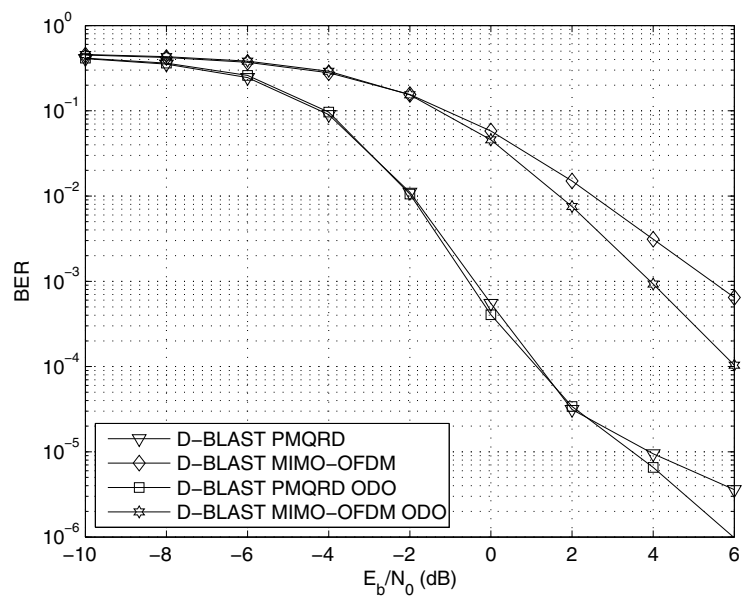

Fig. 4. Average uncoded BER results for D-BLAST PMQRD and MIMOOFDM schemes for a $3 \times 3$ MIMO channel, $L=5$, with constant power profile.

Figure 4 directly compares the proposed PMQRD and MIMO-OFDM QR schemes for both standard and ODO implementations. BER performance of the PMQRD is far superior to the MIMO-OFDM QR scheme, for example a $5 \mathrm{db}$ gain in SNR is observed at BER $10^{-3}$ when ODO is applied.

\section{A. Channel Estimation Errors}

We now consider the scenario where the receiver has imperfect channel knowledge. A training sequence of length $N_{t}, s_{t}(n) \in \mathbb{C}^{M_{t} \times 1}$ for $n \in\left\{0,1, \ldots, N_{t}-1\right\}$ is propagated through the MIMO wireless channel, $\underline{\boldsymbol{H}}(z)$. It is assumed the receiver has prior knowledge of the training sequence. Expressing the received training sequence, $\boldsymbol{y}_{t}(n) \in \mathbb{C}^{N_{t}+L-1, M_{r}}$ as

$$
\boldsymbol{y}_{t}=\boldsymbol{S}_{t} \boldsymbol{H}_{t}+\boldsymbol{n}
$$

where $\boldsymbol{S}_{t}$ is an $N_{t}+L-1 \times M_{t}(L)$ matrix comprised of $N_{t}+L-1 \times(L)$ subblocks, with each subblock representing the convolution matrix of the training sequence transmitted from a given transmit antenna and possessing the well-known Toeplitz form and $\boldsymbol{H}_{t}$ is an $M_{t}(L) \times M_{r}$ matrix containing the channel coefficients $\underline{\boldsymbol{H}}(z)$ in column vector form, i.e.

$$
\boldsymbol{H}_{t}=\left[\begin{array}{ccc}
h_{11}(0) & \ldots & h_{M_{t} 1}(0) \\
h_{11}(1) & \ldots & h_{M_{t} 1}(1) \\
\vdots & & \vdots \\
h_{11}(L-1) & & h_{M_{t} 1}(L-1) \\
\vdots & & \vdots \\
h_{1 M_{r}}(0) & \ldots & h_{M_{t} M_{r}(0)} \\
h_{1 M_{r}}(1) & \ldots & h_{M_{t} M_{r}(1)} \\
\vdots & & \vdots \\
h_{1 M_{r}}(L-1) & \ldots & h_{M_{t} M_{r}(L-1)}
\end{array}\right]
$$

Minimizing the cost function $\left|\boldsymbol{y}_{t}-\boldsymbol{S}_{t} \hat{\boldsymbol{H}}_{t}\right|^{2}$ where $\hat{\boldsymbol{H}}_{t}$ denotes the estimate of the channel matrix is now given by the least squares estimator:

$$
\hat{\boldsymbol{H}}_{t}=\left(\boldsymbol{S}_{t}^{H} \boldsymbol{S}_{t}\right)^{-1} \boldsymbol{S}_{t}^{H} \boldsymbol{y}_{t}
$$

For optimality, the training sequence should be uncorrelated, i.e. $E\left\{\boldsymbol{S}_{t}^{H} \boldsymbol{S}_{t}\right\}=\boldsymbol{I} \times N_{t}$. Therefore we have generated a sequence with a suitably low correlation for use in our simulations. The relative error for the estimate of the channel, $\underline{\boldsymbol{H}}(z)$ is defined as

$$
E_{\text {rel }}=\frac{\|\underline{\boldsymbol{H}} z-\underline{\hat{\boldsymbol{H}}} z\|_{F}}{\|\underline{\boldsymbol{H}} z\|_{F}}
$$

Figure 5 shows the average relative error for 1000 Monte Carlo simulations. Even with a relative low SNR it is possible to obtain a reasonably accurate estimation of the channel, for example a relative error of 0.27 at an SNR of $2 \mathrm{~dB}$.

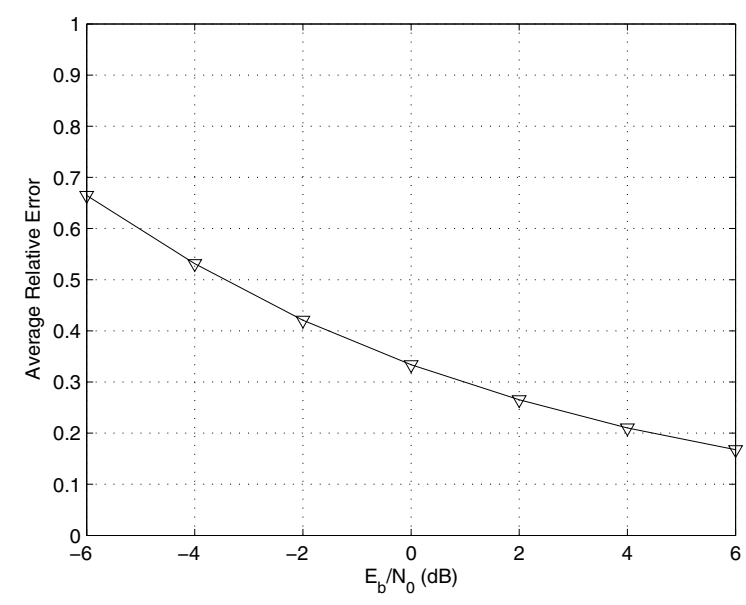

Fig. 5. Average relative error in channel estimation for a $3 \times 3$ MIMO channel $L=5$, with constant power profile and training sequence of length $N_{t}=50$.

Figure 6 shows the impact of channel estimation error on BER performance. Using an identical scheme as previously described we have computed the bit error rate for 1000 Monte Carlo simulations. The modulation scheme used is BPSK. The number of time slots of the channel, $N=2048$. The length 
of the training sequence, $N_{t}=50$. Simulations have been performed for both PMQRD ODO and MIMO-OFDM ODO schemes.

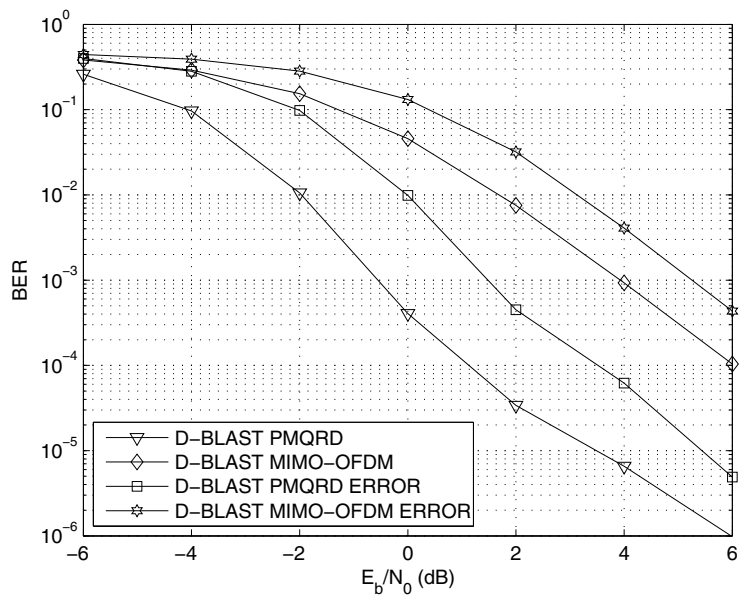

Fig. 6. Average uncoded BER results for D-BLAST PMQRD and MIMOOFDM schemes for a $3 \times 3$ MIMO channel, $L=5$, with constant power profile with channel estimation error. All schemes utilize ODO

Figure 6 again clearly shows the BER performance benefits of the PMQRD ODO scheme. For example a $3.5 \mathrm{~dB}$ gain in SNR is observed at BER $10^{-3}$.

\section{CONCLUSION}

We have proposed a PMQRD technique for MIMO systems with frequency selective channels and implemented a DBLAST architecture based communications system.

For the MIMO-OFDM D-BLAST scheme, the information in each individual symbol, is constrained to a single narrowband tone. Individual tones may have poor gain due to the frequency selective nature of the MIMO channel, resulting in degraded system performance. However in the PMQRD DBLAST based system the information in each symbol is spread across the entire frequency bandwidth, making the system more robust to frequency selectivity, resulting in superior performance. This makes PMQRD highly suitable for MIMOQR based applications where the transmitter has no prior channel knowledge, for example digital video broadcasting.

For modulation schemes with constant transmit energy per symbol, the peak-to-average power ratio (PAR) will be unity for PMQRD based schemes. For an identical OFDM based scheme, the IFFT operation at each transmit antenna results in a relatively high PAR. Therefore, nonlinearities may get overloaded by high signal peaks, causing intermodulation distortion in the transmitted signal [14], and undesired outof-band radiation. If radio frequency (RF) power amplifiers are operated without large power back-offs, it is impossible to keep the out-of-band power below specified limits, leading to very inefficient amplification and expensive transmitters [15]. For modulation schemes with variable transmit energy per symbol, the PAR of PMQRD system will still be significantly less than that of OFDM, reducing transmitter complexity and cost.

In addition, signal components originating from bins other than the considered one give rise to interbin interference (IBI) [16]. In the presence of channel estimation error IBI is significantly increased, leading to degraded performance of an MIMO-OFDM based scheme. Although the PMQRD scheme is also susceptible to channel estimation error, its BER performance is still significantly greater, demonstrating the robustness of the proposed PMQRD scheme.

\section{ACKNOWLEDGEMENT}

The authors would like to thank Dr Ian Prouder for his invaluable comments and suggestions. The work of Martin Davies is jointly funded by EPSRC and QinetiQ. The work of Joanne Foster is funded by EPSRC.

\section{REFERENCES}

[1] G. Golub and C. Loan, Matrix Computations, 3rd ed. Baltimore, Maryland: The John Hopkins University Press, 1996.

[2] A. Paulraj, R. Nabar, and D. Gore, Introduction to Space-Time Wireless Communications, 1st ed. University Press, Cambridge: Cambridge University Press, 2003.

[3] J. Foster, J. McWhirter, and J. Chambers, "A polynomial matrix QR decomposition with application to MIMO channel equalization," in Conference Digest : $41^{\text {st }}$ Asilomar conference on signals, systems, and computers, November 2007.

[4] — "An algorithm for computing the QR decomposition of a polynomial matrix," in $15^{\text {th }}$ International Conference on Digital Signal Processing, Cardiff, July 2007.

[5] M. Tuchler, R. Koetter, and A. Singer, "Turbo equalization: principles and new results," IEEE transactions on communications, vol. 50, no. 5, pp. 754-767, May 2002.

[6] G. Foschini, "Layered space-time architecture for wireless communication in a fading environment when using multi-element antennas," Bell Labs Technical Journal (Autumn), pp. 41-59, 1996.

[7] P. Wolninansky, G. Foschini, G. Golden, and R. Valenzuela, "V-BLAST: An architecture for realizing very high data rates over the rich-scattering wireless channel," in 1998 International Symposium on Signals, Systems and Electronics, Pisa, Sept-Oct 1998, pp. 295-300.

[8] Technical Specification Group GERAN, Channel Coding, 3rd Generation Partnership Project Std. 3GPP TS 05.03 V8.6.1 (2001-01), 1999.

[9] M. Valenti. (2006) Coded modulation library. [Online]. Available: http://www.iterativesolutions.com/Matlab.htm

[10] R. Koetter, A. Singer, and M. Tuchler, "Turbo equalization: an iterative equalization and decoding technique for coded data transmission," IEEE Signal Processing Magazine, pp. 67-80, January 2004.

[11] L. Bahl, J. Cocke, F. Jelinek, and J. Raviv, "Optimal decoding of linear codes for minimizing symbol error rate," IEEE Transactions on Information Theory, vol. 20, pp. 284-287, March 1974.

[12] T. Moon, Error Correction Coding: Mathematical Methods and Algorithms, 1st ed. Hoboken, New Jersey: John Wiley \& Sons, 2005.

[13] B. Vucetic and J. Yuah, Turbo Codes: Principles and Applications, 1st ed. Dordrecht, The Netherlands: Kluwer Academic Publishers, 2000.

[14] J. Proakis, Digital Communications, 4th ed. Avenue of the Americas, New York: McGraw-Hill, 2001.

[15] S. Muller and J. Huber, "A comparison of peak power reduction schemes for OFDM," in Proceedings of IEEE GLOBECOM, Phoenix, Arizona, November 1997, pp. 1-5.

[16] T. Pollet, M. V. Bladel, and M. Moeneclaey, "BER sensitivity of OFDM systems to carrier frequency offset and Wiener phase noise," IEEE Transactions on Communications, vol. 43, no. 2/3/4, pp. 191-193, February 1995. 